\title{
Source Controlled and Delay Sensitive Selective Retransmission Scheme for Multimedia Streaming
}

\author{
Árpád HUSZÁK, Sándor IMRE \\ Budapest University of Technology and Economics, Department of Telecommunications, Mobile \\ Communications and Computing Laboratory, Magyar Tudósok krt.2., H-1117 Budapest, Hungary \\ \{huszak,imre\}@hit.bme.hu
}

\begin{abstract}
Multimedia applications are becoming increasingly popular in IP networks, while in mobile environment the limited bandwidth and the higher error rate arise in spite of its popularity. The wireless network characteristics make the deployment of these applications more challenging.

Retransmission-based error recovery is considered inappropriate for multimedia applications, because of its latency. However, this solution can be attractive because it requires minimal network bandwidth and processing cost as well as improvement of the video quality. Despite its latency, retransmission can be used successfully in many cases, especially if playout buffering is employed.

In this paper we propose a source controlled and playout time oriented retransmission scheme for multimedia streaming. Due to source controlling the receiver do not need to send additional retransmission request messages. The other advantage of transmitter controlled decision algorithm is that all the needed information is available at the source due to DCCP transport protocol. Therefore no additional network measurements and probe messages are needed. The results show that our scheme reduces traffic overhead generated by retransmission and hardly degrades the number of unsuccessfully retransmitted packets at all.
\end{abstract}

\section{Introduction}

The newly appeared applications that rely on the realtime delivery of data, such as video conferencing, Internet telephony, and streaming audio and video players are gaining prominence on the Internet. These applications are not only used in reliable wired networks but also in wireless environment where the obstacles of the expansion are the higher bit error ratio of the radio link and the limited bandwidth of the mobile links.
A packet loss generally degrades the performance of any Internet data transfer especially on compressed data. Inter-frame-video compression algorithms such as MPEG exploit temporal correlation between frames to achieve higher compression therefore errors in a reference frame will propagate to the dependent difference frames. To minimize the end-to-end packet loss ratio the packet loss should be either prevented or subsequently handled.

Traditional error control mechanisms generally use retransmission to provide reliability at the expense of latency. For the retransmission to be successful, retransmitted packet must arrive at the receiver in time for playback. To minimize the probability of wastefully retransmitted packets, a playout buffer is usually set up at the receiver side to prefetch a certain amount of data before playback. The buffered data provides additional time to absorb the retransmission delay making the retransmission acceptable for one-way pre-recorded and one-way live media applications. The key issue is the correct determination of the playout buffer that must be dependent on the network delay to make the retransmission possible.

Loss-tolerant real-time multimedia applications prefer UDP or UDPLite [1] but in our proposal we applied DCCP (Datagram Congestion Control Protocol) [2] as transport protocol because it uses sequence numbering, acknowledgements and congestion control algorithms. Sequence numbers and acknowledgement are needed to identify the lost packets while the congestion control algorithms (TCP-Like Rate Control [3] and TCP Friendly Rate Control [4]) manage the actual Round-Trip Time (RTT) measurements. The actual RTT plays a very important role to decide weather to retransmit a packet. In our scheme the transmitter determines the playout delay caused by the playout buffer using the proposed Flood method.

The rest of the paper is organized as follows. A review of related work in selective retransmission is 
presented in Section II. In Section III we introduce our source controlled selective retransmission method for multimedia applications. The obtained results are presented in Section IV. Finally, we summarize our paper and make the conclusions in the last section.

\section{Related work}

Some prior work has been done to develop error recovery and concealment for real-time video. The method of these works can be divided to content based and network characteristic based selective retransmission algorithms.

\section{A. Content based retransmission}

Content based retransmission methods retransmit only the important data of the bitstream. These approaches describe a method that includes categorizing groups of packets in order of importance. It takes advantage of the motion prediction loop employed in most motion compensation based codecs. Correcting errors in a reference frame caused by earlier packet loss, prevents error propagation.

Feamster and Balakrishnan [5] analyzed this approach with SR-RTP [6]. This RTP extension provides semantics for requesting the retransmission of independently processible portions of the bitstream and a means for reassembling fragmented portions of independently processible units. They have shown that, by recovery of only the most important data in the bitstream, significant performance gains can be achieved without much additional penalty in terms of latency.

In [7] the selective retransmission of MPEG stream was analyzed with DCCP. This transport protocol provides indispensable information to apply semireliable transfer of MPEG video. The results show that the effectiveness of this protocol is considerable when selective retransmission is deployed.

Zheng and Atiquzzaman [8] proposed a new selective retransmission scheme for multimedia transmission over noisy wireless channel using the ATM ABR service. They analyzed the system requirements and minimum receiver buffer size for providing acceptable QoS to the user.

\section{B. Network characteristic based retransmission}

This type of methods investigates the possibility of successful packet retransmissions in the function of the actual network delay and bandwidth. The decision algorithms decide weather to retransmit a packet without late reception.
Attempts were made to implement a selective retransmission protocol with a decision algorithm [9]. This algorithm decides whether or not to request a retransmission for a packet that was detected as lost. The decision is made by the Euclidean distance calculated by the loss and latency ratio. This protocol does not use playout buffer and does not implement congestion avoidance mechanisms.

In the scheme introduced in [10], the server decides whether it retransmits packets or not based not only on importance of each packet but also playout time of each packet. In this proposal when the client detects a packet loss, it sends a retransmission request packet to the server. The request message contains some necessary information to make the retransmission decision successful.

The priority of the two type of retransmission method is not the same. The importance of the network characteristic based method is higher because the successful data transmission is the main goal. The packet content should be taken into consideration only if the possibility of the correct receipt of the retransmitted packet is high enough. It makes no sense to retransmit a high importance packet if it will not arrive in time.

In most of the related works the receiver controls the retransmission procedure. The decision algorithm is implemented at the receiver therefore additional administration messages must be sent to the sender. Most of the prior works use NACK (Negative ACKnowledgement) or Retransmission Request messages. In our proposal no administration messages are needed because the decision procedure is located at the transmitter. The other advantage of the transmitter side decision is that the input parameters of the decision algorithm (RTT, estimated link bandwidth, etc.) are available at the source using the DCCP transport protocol.

\section{Source Controlled and Delay Sensitive Retransmission Scheme}

We propose a selective retransmission scheme based on the DCCP transport protocol. The Datagram Congestion Control Protocol is a newly defined transport protocol by the IETF that implements bidirectional, unicast connections of congestion controlled, unreliable datagrams. For our purpose this protocol is ideal because it provides all the needed information for the decision algorithm. The DCCP header contains sequence number field that is indispensable to recognize and identify the lost packets. DCCP connections are congestion controlled, but unlike 
in TCP, DCCP applications have a choice of congestion control mechanism. Currently two mechanisms are defined: TCP-like Congestion Control, TCP-Friendly Rate Control (TFRC). All kind of congestion control mechanisms need information about the network, like RTT and the packet loss. These are the necessary input for our decision algorithm too.

To make the retransmission possible the receiver must employ a playout buffer while at the DCCP source a retransmission buffer is needed. DCCP does not have a packet retransmission function therefore the server buffers packets transmitted by itself into a retransmission buffer. To measure the elapsed time from the first packet transmission to the detection of loss the transmission time must be stored.

One of the inputs of the decision algorithm is the delay introduced by the playout buffer at the receiver. In our scheme the playout delay is set up by the transmitter using so-called Flood method. To make the decision of retransmission the elapsed time end the actual playout buffer level will be used.

\section{A. Playout Delay Setup with Flood Method}

To make the retransmission controlled by the source without administrative messages the transmitter must know the playout buffer level and its delay. The proposed solution is called Flood method.

At the beginning of the video transmission the transmitter will not deliver the data packets immediately. It will heap up some data and transmit it all together after $T_{b d}$ time as illustrated in Figure 1. With this method the receiver will receive more data then it should process therefore certain amount of data will be heaped up in the receiver's playout buffer. The process rate of the receiver is known by the transmitter because it is equal with the bitrate of the current video so the duration of the Flood method can be calculated easily in theoretical case.

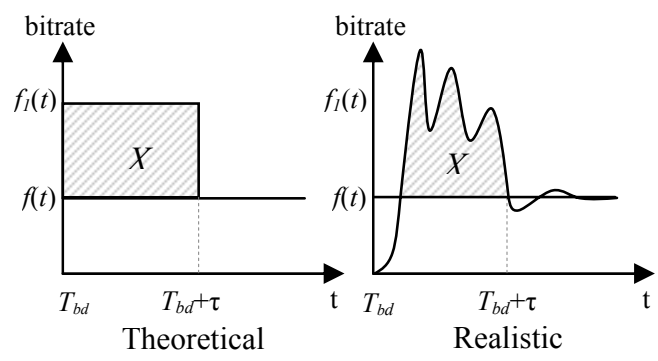

Figure 1. Flood method

The video source birate is $f(t)$ and the increased sending rate of the transmitter during the Flood period is $f_{1}(t)$. In the theoretical case $f_{1}(t)$ is constant during the
$\left[T_{b d}, T_{b d}+\tau\right]$ time interval. The heaped up data can be calculated as follows:

$$
f_{1}(t) \tau-f(t) \tau=X
$$

This extra amount of data $(X)$ will be sent in $\tau$ time.

$$
\tau=\frac{X}{f_{1}(t)-f(t)}
$$

Actually the duration of the Flood method depends on the actual network conditions. In the case of DCCP transport protocol the congestion control algorithm (TFRC) will determine the sending rate during this period therefore $f_{1}(t)$ is not constant. When all the heaped up data is delivered the sending rate will be similar to the bitrate of the video stream and the receiver's playout buffer will contain $X$ amount of data. The time that a data packet will spend in the playout buffer is $T_{b d}$ that is equal with the heap up time before the transmission.

$$
X=T_{b d}(t) \cdot f(t)
$$

We assume that the video bitrate $f(t)$ is constant therefore the buffer delay $T_{b d}(t)$ will not vary intensively. With this method the playout delay is set up by the transmitter so one of the input data for the retransmission decision algorithm is given.

The arising question is that how to determine the $X$ to introduce adequate delay for retransmissions. The playout buffer delay should be long enough to make the packet loss detection and the retransmission feasible but it should be as short as possible. To answer this question first the decision algorithm must be reviewed.

\section{B. Decision Algorithm}

The decision algorithm will decide weather to retransmit a packet or not. The algorithm will work properly if all the retransmitted packets arrive in time and there is no lost packet that was not retransmitted although it would arrive before the playout.

The available time for the first transmission, retransmission and loss detection is equal with the playout buffer delay.

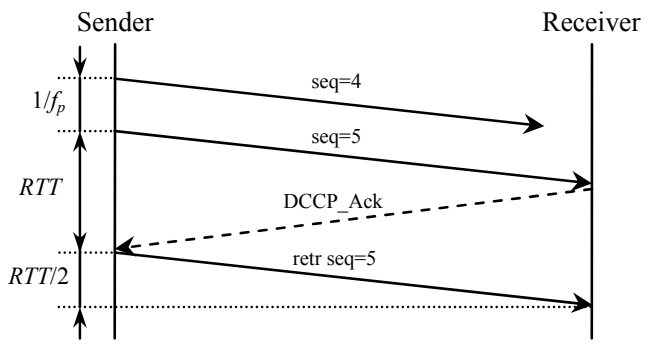

Figure 2. Transmission sequence 
Figure 2. depicts a transmission and a retransmission with gap detection to detect the lost packets. In this situation the retransmission is enabled if

$$
\frac{3}{2} R T T+\frac{1}{f_{p}}<T_{b d},
$$

where $f_{p}$ is the frequency of packet sending so the elapsed time between two packets is $1 / f_{p}$.

In general the stipulation to successfully retransmit a packet is given in (5).

$$
\frac{3}{2} R T T+\delta<T_{b d}
$$

where $\delta$ is the loss detection delay.

The upper limit of the loss detection delay is determined by the congestion control algorithm because this algorithm specifies the frequency of the DCCP acknowledgements. The receiver sends DCCP-Ack packets at least once per Round-Trip-Time acknowledging the data packets, unless the sender is sending at a rate of less than one packet per RTT, as indicated by the TFRC specification [RFC 3448]. According to the specification the loss detection delay is

$$
\frac{1}{f_{p}}<\delta<R T T .
$$

The actual value of the loss detection delay varies therefore the packet sending time $\left(t_{1}\right)$ and the acknowledgement reception time $\left(t_{0}\right)$ is used to determine the elapsed time.

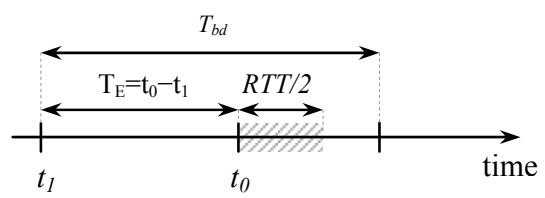

Figure 3. Time sequence

After receiving an acknowledgement, that contains the sequence numbers of lost packets, the transmitter should decide which packet is worth to retransmit. The decision algorithm calculates the remaining time that must be less then the one-way network delay for successful retransmission.

$$
\left(t_{0}-t_{1}\right)-T_{b d}<R T T
$$

\section{Determination of the Playout Buffer Delay}

The way of adjusting the playout buffer delay is previously introduced in Section III/A. but the appropriate value of this parameter is not determined yet. The limitations of the playout buffer delay $\left(T_{b d}\right)$ are determined by the streaming application and the network delay. The upper bound of $T_{b d}$ is the maximal acceptable delay that is few seconds in the case of one-way live media applications but it should not be higher then 150$200 \mathrm{~ms}$ for two-way interactive applications. In the retransmission point of view the lower bound of the playout buffer delay is determined by the network delay. Our goal is to find lowest delay possible to make the retransmission possible.

According to (5) and (6) the upper bound of the sum of the time needed for the transmission and the time needed for the loss detection is

$$
\frac{3 R T T}{2}+\delta \leq \frac{3 R T T}{2}+R T T=2.5 R T T
$$

Several researchers have analyzed the characteristic of the RTT so far. The Round-Trip-Time is usually modeled with heavy-tailed Gamma [11] or with normal distribution [12]. We used the normal distribution model $N\left(\mu, \sigma^{2}\right)$ where $\mu=R T T$ and $\sigma^{2}=(0.1 R T T)^{2}$ to determine the playout buffer delay in our retransmission scheme. We have determined the playout buffer delay in such a way that the probability of successful retransmission should be higher then $95 \%$.

The normalized probability density function is given in (9), where $u=\frac{X-\mu}{\sigma}$.

$$
\begin{gathered}
\Phi(x)=\frac{1}{\sqrt{2 \pi}} \int_{-\infty}^{x} \exp \left(-\frac{u^{2}}{2}\right) d u=0.95 \Rightarrow x=1.65 \\
X=\mu+x \sigma=2.91 R T T \approx 3 R T T
\end{gathered}
$$

In our retransmission scheme we propose to set the playout buffer delay to $T_{b d}=3 R T T_{0}$, where $R T T_{0}$ is the first RTT measured between the source and destination. If the RTT is too high the $T_{b d}$ should be set to the highest acceptable delay depending to the application demands. According to the calculated playout buffer delay the amount of the heaped data can be determined as given in (3).

\section{Simulation results}

In order to test the performance of the selective retransmission scheme, described in the previous section, we analyzed some scenarios with ns-2 [13] network simulator. The simulation environment made it possible to adjust the link characteristics and analyze the effects of additional background traffic. We used a constant bitrate video stream in the simulations but of course our method is applicable for variable bitrate streams too. In the case of variable bitrate streams the average coding rate must be known.

In the first scenario we have analyzed the retransmission probability of the lost packets. The packet loss probability was set to $1 \%$ while the network delay was varied between $10 \mathrm{~ms}$ and $150 \mathrm{~ms}$; the video stream 
bitrate was $384 \mathrm{kbps}$. During the 150 s simulation time about 5000 packet was sent. The packet size was 1500 bytes.

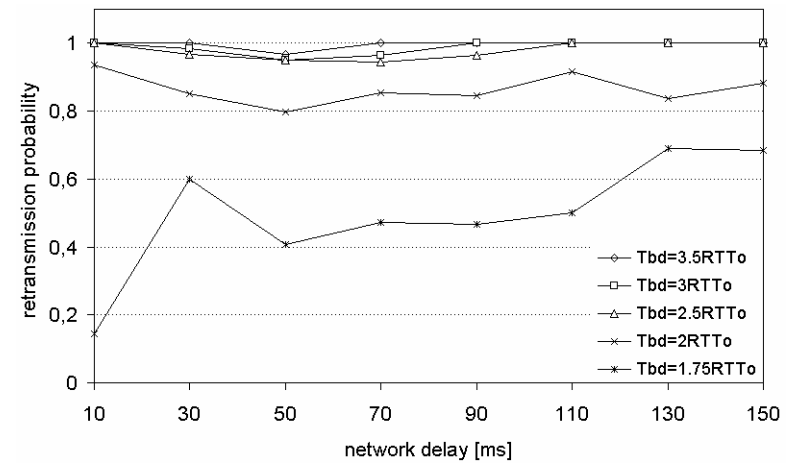

Figure 4. Retransmission probability

On ratio of retransmitted packets to all lost packets, we compare different playout buffer delay setups. The result show that with $T_{b d}=3 R T T$ most of the lost packets can be retransmitted.

Examinations were made to find the limits of the playout buffer delay in the retransmission point of view. The next figure shows the maximal playout buffer delay when no retransmission is possible and the minimal playout buffer delay when all the packets can be retransmitted.

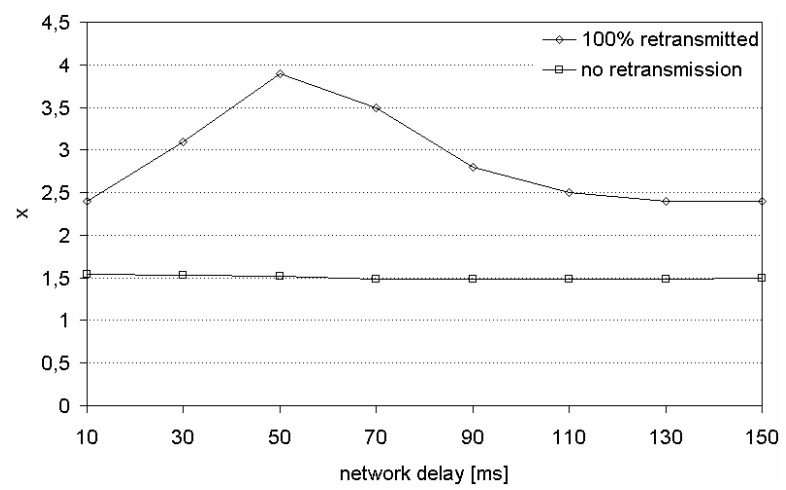

Figure 5. Retransmission probability, $T_{b d}=x R T T_{0}$

The lower bound of $T_{b d}$ is $1.5 R T T_{0}$. In the simulations there were always some packets that were detected as lost in short time. The other reason of receiving some retransmitted packets in near $1.5 R T T_{0}$ time is that the $T_{b d}$ is defined by the RTT at the beginning of the transmission. If the initial RTT is higher than the actual, some retransmission should be enabled if $T_{b d}=1.5 R T T_{0}$.

The main goal of our proposal is to improve the quality of video streams. To see the effectiveness our source controlled selective retransmission scheme we measure the average peak signal-to-noise ratio (PSNR) of the transmitted video stream with different $T_{b d}$ settings. PSNR values measured against the original frames with varying degrees of packet loss. As the packet loss increases the frame-by-frame PSNR drops dramatically. In the PSNR measurements the network delay was $90 \mathrm{~ms}$.

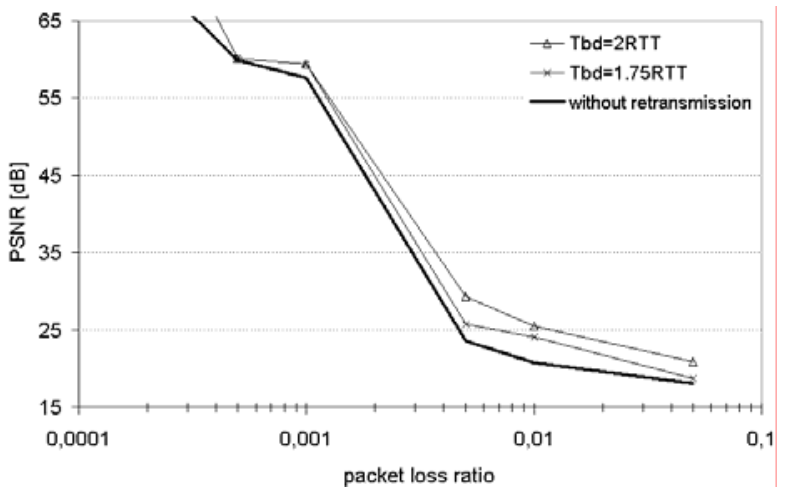

Figure 6. Average PSNR of 150 seconds of $24 \mathrm{fps}$ video as a function of packet loss

We propose to set the playout buffer delay to $3 R T T_{0}$ because in this case almost all of the lost packets can be retransmitted; therefore the measured average PSNR value is similar to the original video stream. The video quality improvement with $T_{b d}=2 R T T_{0}$ is between $2-3 \mathrm{~dB}$ while in the case of $T_{b d}=1.75 R T T_{0}$ it was $1-2 \mathrm{~dB}$. The frame-by-frame PSNR comparison of the video stream with retransmission $\left(T_{b d}=1.75 R T T_{0}\right)$ and without retransmission is illustrated on the next figure.

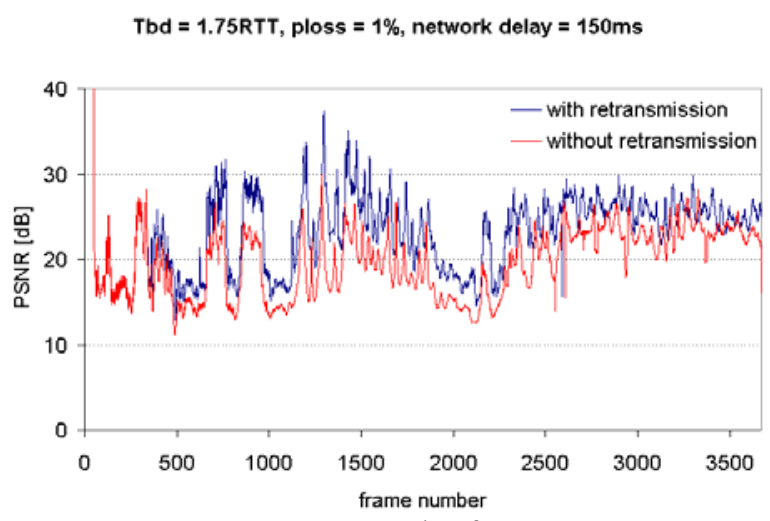

Figure 7. Frame-by-frame PSNR

The acceptable playout buffer delay is limited by the type of the streaming application. In some situations the $T_{b d}$ should not set to $3 R T T_{0}$ but it is worth to set to its maximal acceptable value. Figure 7. shows that even with $T_{b d}=1.75 R T T_{0}$ quality improvement can be achieved.

To obtain these results no additional messages were needed, because the DCCP transport protocol has provided all the necessary information for the Flood method and for the decision algorithm. Even more the 
DCCP employs congestion control mechanisms so this is another advantage of our solution.

\section{Conclusion}

The increase of multimedia applications over the Internet has placed new requirements on current Internet protocols. These applications can tolerate small amounts of data loss but with retransmissions the loss ratio can be minimized improving the quality of the video stream. In live multimedia applications the retransmission can be realized with playout buffer deployed at the receiver.

In this paper we proposed a new source controlled retransmission scheme with a decision algorithm. The main advantage of the proposed source controlled mechanism is that all the needed input parameters of the decision algorithm are available at the transmitter side without any additional administrative messages. We also defined the sufficient playout buffer delay to make the retransmission suitable for the proposed scheme.

To evaluate our proposal simulations were performed and the obtained results proved the efficiency of our retransmission scheme.

The algorithm for deciding whether or not to retransmit a lost packet, adapts itself not only to each application, but also to alternate network conditions. Thus, the flexibility and performance of the source controlled selective retransmission scheme, provides a potential framework for Internet multimedia applications to achieve better quality.

\section{Acknowledgement}

This work was supported by the ETIK (InterUniversity Cooperative Research Centre for Telecommunications \& Informatics), the Mobile Innovation Centre (MIK) and the ANEMONE project which is partly funded by the Sixth Framework Programme of the European Commission's Information Society Technology.

\section{References}

[1] Larzon, Degermark, Pink, "The Lightweight User Datagram Protocol", Internet Engineering Task Force, RFC 3828, July 2004.

[2] Kohler, Handley, Floyd, "Datagram Congestion Control Protocol”, Internet Engineering Task Force, RFC 4340, March 2006.

[3] S. Floyd and E. Kohler, "Profile for DCCP Congestion Control ID 2: TCP-like Congestion Control", draft-ietf-dccpccid2-10.txt, March 2005.

[4] S. Floyd, E. Kohler, and J. Padhye, "Profile for DCCP Congestion Control ID 3: TFRC Congestion Control", draftietf-dccp-ccid3-11.txt, March 2005.

[5] M. Feamster, H. Balakrishnan, "Packet Loss Recovery for Streaming Video", 12th International Packet Video Workshop, Pittsburgh, PA, April 2002.

[6] A. Miyazaki, A. H. Fukushima, K. Hata, T. Wiebke, R. Hakenberg, C. Burmeister, Matsushita, "RTP payload formats to enable multiple selective retransmission", Internet Engineering Task Force, draft-ietfavt-rtp-selret-04.txt, Nov. 2001.

[7] A. Huszak, S. Imre," Selective Retransmission of MPEG Video Streams over IP Networks", CSNDSP 2006, Patras, Greece, July 2006.

[8] Bing Zheng, Mohammed Atiquzzaman, "Network Requirement for Management of Multimedia over Wireless Channel", Lecture Notes In Computer Science Vol. 2496, Proceedings of the 5th IFIP/IEEE International Conference on Management of Multimedia Networks and Services: Management of Multimedia on the Internet, London, UK, 2002.

[9] Mike Piecuch, Ken French, George Oprica, Mark Claypool, "A Selective Retransmission Protocol for Multimedia on the Internet", Proceedings of SPIE International Symposium on Multimedia Systems and Applications, Boston, MA, USA November 2000.

[10] H. Hagino, Y. Miyazaki, Y. Onoe, Y. Atsumi, H. Komaki, M. Taniguchi, N. Yamanouchi, "A Playout Time Oriented Retransmission Scheme for Multimedia Streaming Systems", HSNMC 2003, Estoril, Portugal, 23-25 July 2003., pp406-415

[11] Mark Kalman, Bernd Girod, "Modeling the Delays of Successively-Transmitted Internet Packets", IEEE Conference on Multimedia and Expo, Taipei, Taiwan, June 2004.

[12] Yasuhiro Sato, Shingo Ata, Ikuo Oka, Chikato Fujiwara, "Using Mixed Distribution for Modeling End-to-End Delay Characteristics", Proceedings of the 8th Asia-Pacific Network and Management Symposium (APNOMS 2005), Japan, Okinawa, September 2005.

[13] Ns-2 - Network Simulator, http:///www.isi.edu/nsnam/ns/index.html 